\title{
Supramolecular Engineering Of Metal-Organic Networks At Surfaces
}

\author{
Nian Lin*, Alexandre Dmitriev*, Hannes Spillmann*, Paolo Messina*, \\ Magali Lingenfelder*, Sebastian Stepanow*, Johannes V. Barth ${ }^{\dagger}$ and \\ Klaus Kern *,† \\ * Max-Planck-Institut für Festkörperforschung, D-70569 Stuttgart \\ ${ }^{\dagger}$ Dept. of Institut de Physique des Nanostructures, Ecole Polytechnique Fédérale de Lausanne, \\ CH-1015 Lausanne, Switzerland
}

\begin{abstract}
We report the construction of complex metal-organic assemblies at surfaces using concepts from coordination chemistry. ${ }^{1}$ Well-ordered supramolecular assemblies and metalorganic coordination networks with specific topologies and a high structural stability have been fabricated under ultra-high vacuum conditions by sequential deposition of polyfunctional organic molecules and $\mathrm{Fe}$ atoms onto an atomically clean $\mathrm{Cu}(100)$ substrate. ${ }^{2}$ The structures were investigated at the molecular level by in-situ scanning tunneling microscopy. The precise control of the concentration ratio of the components and the annealing treatment allow for the assembly of distinct architectures.
\end{abstract}

\section{INTRODUCTION}

Metal-organic coordination networks (MOCNs) have been realized in threedimensional bulk materials. ${ }^{3-8}$ The synthesis has been directed by the rational design principle, i. e., assembling well-defined and rigid molecular building blocks into predesigned ordered structures. These materials have gained wide attentions because of their high stability, tunable dimensions, porosity and chemical functionality. ${ }^{9}$ They may find various applications in molecular recognition, heterogeneous asymmetric catalysis, inclusion phenomena, etc. ${ }^{10-14}$ For many applications the controlled nanoscale fabrication of functional molecular architectures at substrates is desirable. ${ }^{15-}$

17 However, most of the molecular assemblies at surfaces reported to date are stabilized by relatively weak interactions, such as hydrogen bonding, van der Waals or electrostatic forces, implying a limited thermal stability. ${ }^{18-20}$ The metal-organic coordination systems, which represent more robust structures, have not been exploited at surfaces so far.

Here we expound a new strategy to fabricate surface-supported MOCNs at a welldefined metal surface under ultra-high vacuum (UHV) conditions. The sequential deposition of polyfunctional organic molecules and $\mathrm{Fe}$ atoms on an atomically clean $\mathrm{Cu}(100)$ substrate gave rise to the formation of two-dimensional surface-supported open networks, stabilized by relatively strong lateral metal-organic coordination bonds. The structures of the MOCNs were characterized at the molecular level by

CP696, Scanning Tunneling Microscopy/Spectroscopy and Related Techniques: 12th International Conf., edited by P. M. Koenraad and M. Kemerink

(C) 2003 American Institute of Physics 0-7354-0168-3/03/\$20.00 
scanning tunneling microscopy (STM). We have discovered that by tuning of the molecule to $\mathrm{Fe}$ concentration ratio and altering annealing parameters, distinct architectures may be produced. Due to the specific chemistry of the molecules and metal atoms, these structures may bestow intriguing functionality to the surfaces. ${ }^{21-23}$ This approach inherits the merits of the rational design principle for bulk MOCN materials and opens up new possibilities for the bottom-up fabrication of lowdimensional functional materials with potential applications in data storage, asymmetric catalysis, molecular recognition hosts and functional nanotemplates.

\section{EXPERIMENTS}

The organic molecules and iron atoms were sequentially deposited on the (100) surface of a $\mathrm{Cu}$ single crystal in a standard UHV chamber with a base pressure of $\sim 3 \times 10^{-10}$ mbar. The ultra-high vacuum environment ensures a clean synthesis condition that excludes contaminations from dust and ambient gases. The $\mathrm{Cu}(100)$ surface was cleaned by repeated cycles of $\mathrm{Ar}^{+}$sputtering and subsequent annealing to $800 \mathrm{~K}$, whereupon flat terraces of up to $50 \mathrm{~nm}$ width separated by monatomic steps were obtained.
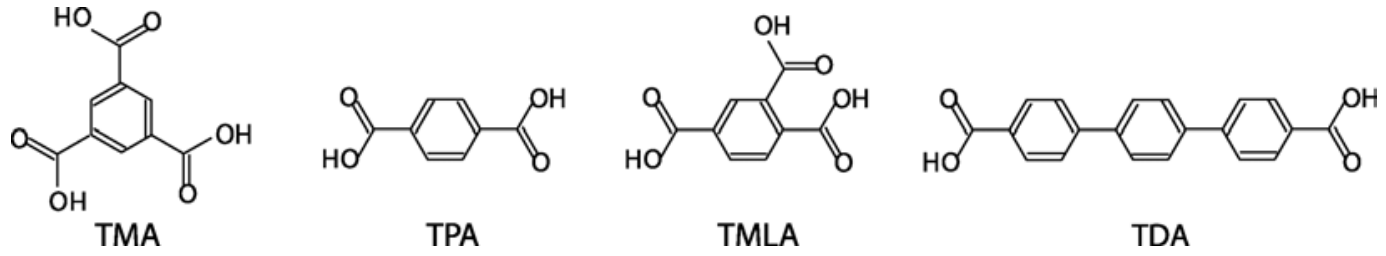

SCHEME 1. Polyfunctional organic molecules used in the present study.

As shown in the scheme 1, the organic molecules used in the present study are 1,3,5-tricarboxylic benzoic acid (trimesic acid, TMA), 1,4-benzenedicarboxylic acid (terephthalic acid, TPA), 1,2,4-benzenetricarboxylic acid molecules (trimellitic acid, TMLA) and 4,1',4',1"-terphenyl-1,4"-dicarboxylic acid (TDA). TMA, TPA and TMLA have an aromatic ring (three aromatic rings for TDA) as backbone and all four have carboxylic groups as functional endgroups. The molecules were sublimated by organic molecular beam epitaxy (OMBE) from Knudsen-cell type evaporators and deposited at the $\mathrm{Cu}(100)$ surface. At the temperature employed in the experiments, deprotonation of the carboxylic groups of the surface-adsorbed molecules is expected to occur, which provide carboxylate groups readily for metal coordination. Fe atoms were evaporated using an e-beam heating evaporator. The temperature of the $\mathrm{Cu}(100)$ surface was controlled by cooling/heating stage, both during and after the depositions, to alter the reaction pathway and to reach the thermodynamic equilibrium. Two strategies have been employed to construct the surface-supported MOCNs: (1) synthesize simple metal-organic compounds at the surfaces first and then let them selfassemble into networks via hydrogen-bond at higher temperatures; (2) first prepare ordered organic molecular overlayer on the surfaces and then introduce metal centers to achieve extended networks. In both cases annealing at $400 \mathrm{~K}$ was performed to 
increase mobility and reactivity of the adsorbates and thus to allow for the formation of well-ordered structures. STM experiments employing the constant current mode were subsequently performed in situ following cool-down to room temperature. The MOCNs were found to be robust, with their morphology being maintained up to a temperature range of 450-500 K.

\section{RESULTS AND DISCUSSION}

\section{(I) Modify The Achiral Surfaces By Homochiral MOCNs}

The TMA molecules and Fe atoms assemble in the form of mononuclear compounds at the $\mathrm{Cu}(100)$ surface, which are stabilized by metal-ligand coordination interactions (Fig. 1a). They represent surface-supported Fe-carboxylates compounds with four molecular ligands coordinated in a square-planar fashion by the central transition metal atom. However, it is important to note that the Fe(TMA) $)_{4}$ complex cannot be regarded as an isolated entity as the coordination bonding and the electronic states of both Fe and TMA are affected by the presence of the metal surface electrons effectively screening charged adsorbates. Thus an isolated deprotonated TMA molecule should not be considered as $\left(\mathrm{TMA}_{\mathrm{ad}}\right)^{3-}$, but rather as neutral TMA/Cu(100) configuration. This issue has to be considered also for all later cases.

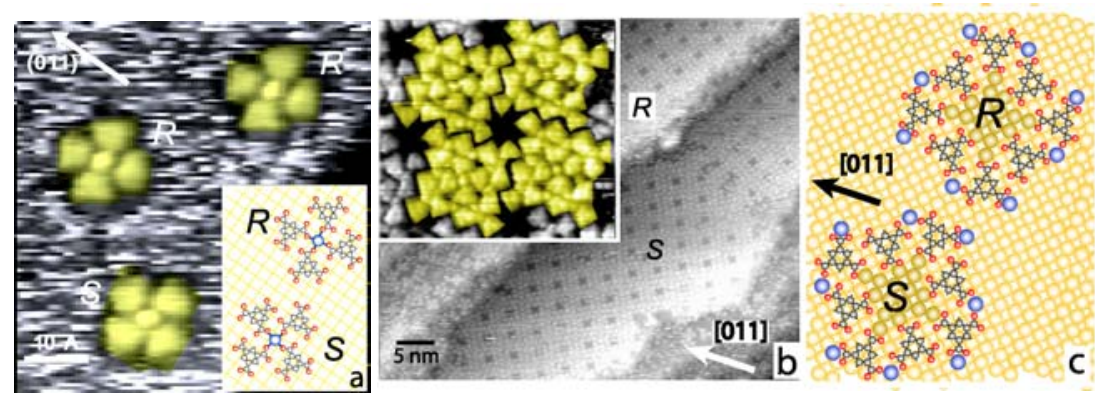

FIGURE 1. (a) Chiral metal-organic compounds at surfaces. (b) Chiral resolution by self-assembly of the chiral compounds, giving rise to the homochiral domains with nanocavities (black dots in the image). (c) Model of the chiral nanocavities in the homochiral domains of (b).

Due to the steric restriction, four TMA molecules bind the central Fe universally via left (or right) oxygen atoms of their carboxylato groups in each compound. As a consequence the two species resulting from left or right oxygen bonding cannot be imposed onto each other by translation or rotation at the surface, i.e., they are enantiomers of the surface chiral compounds. Two enantiomeric phases coexist and equally distribute on the surface, representing a racemic phase. We discovered that the chiral resolution of the racemic mixture could be achieved by the self-assembly of the chiral compounds at higher temperatures. First the chiral compounds turned to polynuclear nanogrids representing the dissymmetric motifs at $350 \mathrm{~K}$ (the inset of Fig. 1b). Then an annealing at $400 \mathrm{~K}$ leads to the organization of mesoscale networks, presumably stabilized via hydrogen bond, as shown in Fig. 1b. The STM topography 
implies that the self-assembly is a chiral recognition and separation process since at the final stage only the enantiomeric species arrange with each other into large homochiral domains. A detailed investigation demonstrates that the arrangement of the nanogrids (colored in yellow in the inset of Fig. 1b) follows a P4 symmetry group. The domains comprise a regular arrangement of nanocavities, which present identically shaped $\sim 1 \mathrm{~nm}$ diameter cavities, equally spaced by $3.43 \mathrm{~nm}$ from each other in both perpendicular directions.

Since each TMA vertex carries a carboxylate group, in total eight COO ligands surround a single cavity. A tentative model of the nanocavities is shown in Fig. 1c (the orientation of the carboxylate groups cannot be conclusively determined on the basis of STM data, and they may rotate out of the molecular plane). The distinct size, shape and chemical functionality make the nanocavity arrays a promising candidate to be employed as host system for the selective adsorption of molecular guests. In addition to the chemical functionality of the cavities, the underlying $\mathrm{Cu}$ substrate exposed by the nanocavities of the $\mathrm{R}$ domains is a mirror image to those of $\mathrm{S}$ domains. Therefore the nanocavities also contain chiral information, which is of great interest for enantioselective recognition and asymmetric catalysis.

\section{(II) Design MOCNs Of Specific Topologies, Functions And Dimensions}

The linear molecules of terephthalic acid (TPA) also react with $\mathrm{Fe}$ at the $\mathrm{Cu}(100)$ surface and form distinct architectures. The TPA to Fe ratios can tune the topology of the architectures. At small Fe concentrations flower-shaped complexes (Fig. 2a) are produced, resembling the $\mathrm{Fe}(\mathrm{TMA})_{4}$ compounds. These complexes assemble as wellordered extended arrays, presumably via hydrogen bonds, with a (6x6) unit cell respect to the $\mathrm{Cu}(100)$ substrate. It represents a 2-dimensional system consisting of periodically arranged individual magnetic centers that are separated from each other by $1.5 \mathrm{~nm}$. At higher Fe dosing we observed different types of network architectures that depend on the subtle control of the Fe concentration. Fig. 2b-d depict the topology of these networks, formed with increasing Fe ratio. Different networks present the open cavities of different sizes. Based on the STM topography the structures of the MOCNs are rationalized by the models in Fig. 2, where Fe centers are coordinated by the oxygen of the carboxylate groups in a plane square manner.
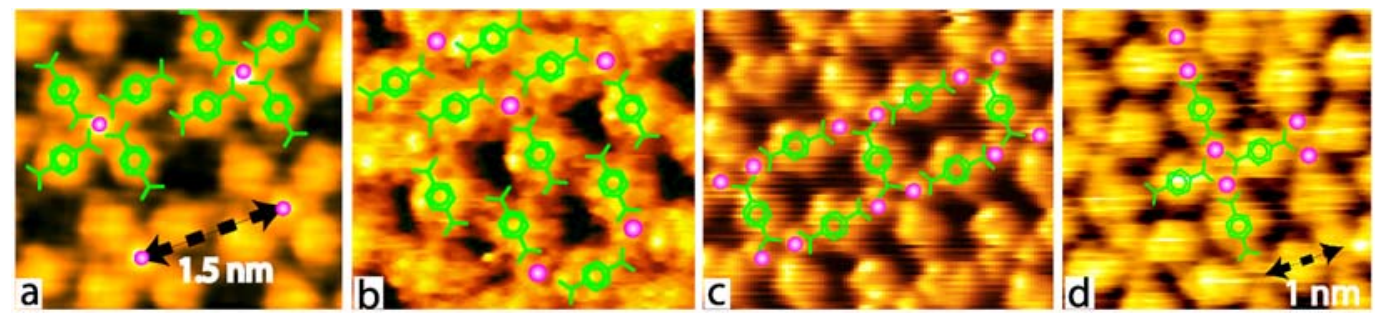

FIGURE 2. STM topography of the metal-organic structures synthesized at the $\mathrm{Cu}(100)$ surface at different TPA/Fe ratios. (a) Ordered arrays of flower-shaped complexes; (b-d) Networks of distinctive topologies formed with increasing Fe concentration. The tentative models show the proposed structures, where TPA molecules are shown as green and Fe atoms in pink. 
It is interesting to address some of possible applications of the networks. Firstly, the periodically arranged magnetic atoms that are mediated by organic linkers represent a potential nanoscale molecule-based magnetic system. Secondly, the obtained MOCNs comprise cavities of well-defined geometry that expose the $\mathrm{Cu}$ substrate. Thus the present approach provides a means to deliberately divide up a surface at the molecular scale. Since the chemical properties of the cavities are furthermore expected to depend on both the linker molecules and node atoms, they can be regarded as nanoscale hosts or even potential nanoreactors providing a well-defined local environment.

These two-dimensional structures can be further functionalized by using molecules that contain additional side groups with specific chemical functions. For instance, similar network structures have been reproduced from 1,2,4-benzenetricarboxylic acid molecules (TMLA) which have one more carboxylic group at o-position, ${ }^{20}$ as shown in Fig. 3a. Therefore the cavities formed here are modified by free carboxyl groups, which would alter the chemical properties of the cavities. We also succeeded in the assembly of a 2-D MOCN which can be regarded as a higher analogue to the TPA MOCNs by using the longer molecule 4,1',4',1"-terphenyl-1,4"-dicarboxylic acid (TDA). This is demonstrated by the STM data in Fig. 3b, clearly the reticulation of the components in an extended network with large cavities reflecting the increased length of the employed linker molecule. It is easy to recognize how the TDA functional endgroups are pointing to the network intersections where metal-ligand interactions are operative.

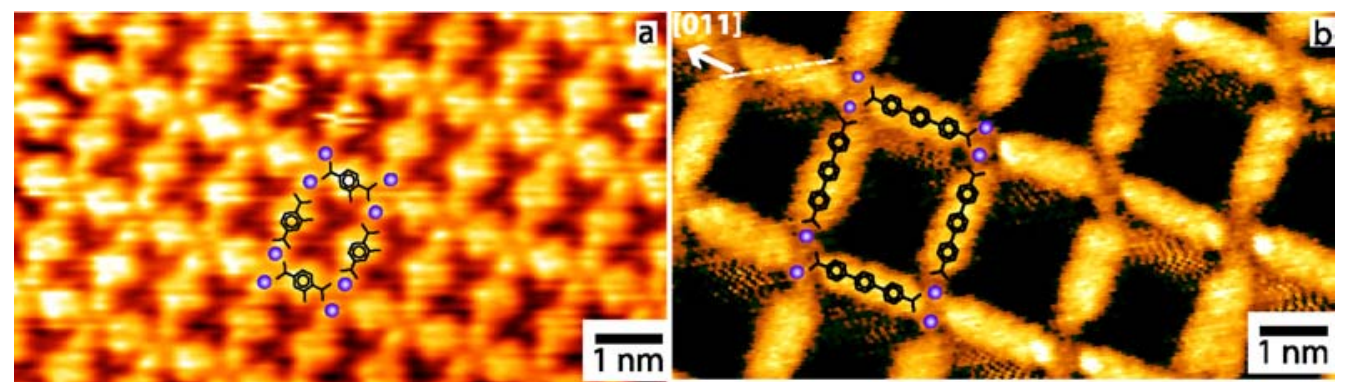

FIGURE 3. MOCN with special functionality and dimension. (a) The TMLA-Fe MOCN: additional carboxylic groups at the cavities; (b) The TDA-Fe MOCN: larger cavities.

\section{CONCLUSION AND OUTLOOK}

We have demonstrated the successful realization of metal-organic coordination networks at the metal surfaces, which opens up a novel route to functionalize surfaces rationally. By selecting proper molecules and suitable synthesis recipes, pre-designed complicated 2-dimensional structures can be fabricated in a controllable way.

Future is even brighter considering the possibilities of using (1) other available organic molecules; (2) alternative metal elements in the periodic table and (3) substrates of different symmetries and different materials (metals, semiconductors and 
insulators). The immense wealth and scope of synthesizing metal-organic networks directly from simple building units at surfaces is apparent.

\section{ACKNOWLEDGMENTS}

We are grateful that Prof. C. Cai of Univeryity of Houston provided the TDA molecules.

\section{REFERENCES}

1. Lin, N., Dmitriev, A., Weckesser, J., Barth, J. V. and Kern, K., Angew. Chem. Int. Ed. 24, 4779 (2002).

2. Messina, P., Dmitriev, A., Lin, N., Spillmann, H., Abel, M., Barth, J.V. and Kern, K., J. Am. Chem. Soc. 124, $14000(2002)$

3. Stang, P. J., Persky, N. E. and Manna, J. , J. Am. Chem. Soc, 119, 4777 (1997).

4. MacGillivray, L. R., Groeneman, R. H. and Atwood, J. L., J. Am. Chem. Soc. 120, 2676 (1998).

5. Batten, S. R. and Robson, R., Angew. Chem. Int. Ed. (37), 1460 (1998).

6. Moulton, B. and Zaworotko, M. J., Chem. Rev. 101, 1629 (2001).

7. Eddaoudi, M., Moler, D. B., Li, H., Chen, B., Reineke, T. M., O'Keeffe, M. and Yaghi, O. M. Acc. Chem. Res, 34, 319 (2001)

8. Cui, Y., Evans, O. R., Ngo, H. L., White, P. S. and Lin, W., Angew. Chem. Int. Ed. 41, 1159 (2002).

9. Yaghi, O. M., O’Keeffe, M., Ockwig, N. W., Chae, H. K., Eddaoudi, M. and Kim, J., Nature, 423, 705 (2003).

10. Li, H., Eddaoudi, M., O'Keeffe, M. and Yaghi, O. M., Nature, 402, 276 (1999).

11. Seo, J. S., Whang, D., Lee, H., Jun, S. I., Oh, J., Jeon, Y. J. and Kim, K., Nature, 404, 982 (2000).

12. Gardner, G. B., Venkataraman, D., Moore, J. S. and Lee, S., Nature, 374, 792 (1995).

13. Yaghi, O. M., Li, G. and Li, H., Nature, 378, 703 (1995).

14. Kepert, C. J., Prior, T. J. and Rosseinsky, J., J. Am. Chem. Soc., 122, 5158 (2000).

15. Collier, C. P., Wong, E. W., Belohradský, M., Raymo, F. M., Stoddart, J. F., Kuekes, P. J., Williams, R. S. and Heath, J. R., Science, 285, 391 (1999).

16. Lopinski, G. P., Wayner, D. D. M. and Wolkow, R. A. , Nature, 406, 482 (2000).

17. Joachim, C., Gimzewski, J. K. and Aviram, A., Nature, 408, 541 (2000).

18. Yokoyama, T., Yokoyama, S., Kamikado, T., Okuno, Y. and Mashiko, S., Nature, 413, 619 (2001).

19. Barth, J. V., Weckesser, J., Cai, C., Günter, P., Bürgi, L., Jeandupeux, O. and Kern, K., Angew. Chem. Int. Ed, 39, 1230 (2000);

20. Böhringer, M., Morgenstern, K., Schneider, W.-D., Berndt, R., Mauri, F., Vita, A. De and R. Car, Phys. Rev. Lett., 83, 324 (1999).

21. Dmitriev, A., Spillmann, H., Lin, N., Barth, J.V. and Kern, K., Angew. Chem. Int. Ed, 42, 2670 (2003).

22. Spillmann, H., Dmitriev, A., Lin, N., Messina, P., Barth, J.V. and Kern, K., submitted.

23. Stepanow, S., Lingenfelder, M., Dmitriev, A., Spillmann, H., Delvigne, E., Lin, N., Deng, X., Cai,C., Barth, J. V. and Kern, K., submitted.

24. Fasel, R., Agostino, R. G., Aebi, P. and Schlapbach, L., Phys. Rev. B., 60, 4517 (1999). 